\title{
Effect of axillary brachial plexus blockade on baroreflex-induced skin vasomotor responses: assessing the effectiveness of sympathetic blockade
}

\author{
T. SzILi-ToroK ${ }^{1}$, D. PAPriKa ${ }^{1}$, Z. Peto ${ }^{2}$, B. BABIK ${ }^{3}$, F. BARI ${ }^{4}$, P. BARzO ${ }^{5}$ and L. Rudas ${ }^{1}$ \\ Departments of ${ }^{1}$ Medical Intensive Care Unit, ${ }^{2}$ Anesthesiology, ${ }^{3}$ Heart Surgery, ${ }^{4}$ Physiology and ${ }^{5}$ Neurosurgery, Albert Szent-Györgyi Medical \\ University, Szeged, Hungary
}

\begin{abstract}
Background: The combination of laser Doppler flowmetry and non-invasive blood pressure monitoring allows the continuous observation of cutaneous vascular resistance (CVR). Continuous recording of unmodulated skin blood flow (SBF) is very sensitive to artefacts, rendering the method unreliable. In contrast, intermittent short lasting challenges of the CVR by cardiovascular autonomic reflexes may provide information about the responsiveness of the sympathetic nervous system in the skin.

Methods: Eleven patients with below-wrist hand surgery (six males and five females; aged 35.2 \pm 7.1 years) performed Valsalva maneuver following axillary blockade. Skin blood flow was continuously monitored on the forearm of the side axillary blockade, as well as on the contra-lateral forearm, which was used as the control. The responses were expressed as changes compared with the baseline level derived from a resting period of $30 \mathrm{~s}$. The maximal change in CVR was determined during the late strain phase of the Valsalva maneuver on both sides. For numerical comparison the change in CVR on the axillary blockade and control sides were simultaneously calculated.
\end{abstract}

Results: During the Valsalva maneuver a significant increase in
CVR was observed on the control side with a maximum value during the late strain phase (baseline $0.18 \pm 0.1$ and late strain phase $0.42 \pm 0.2$ relative units; $P<0.01$ ). In contrast, only minimal changes were detected on the side of axillary blockade in CVR (baseline $0.17 \pm 0.8$ and late strain $0.16 \pm 0.2$ relative units; $P=\mathrm{NS}$ ). Conclusions: Our findings support the disputed hypothesis that the human skin microvasculature is involved in baroreflex regulation under thermoneutral conditions. The determination of baroreflex stimulus-induced microvascular responses may serve as a feasible method for monitoring the effectiveness of sympathetic blockade.

Received 29 March 2001, accepted for publication 20 February 2002

Key words: laser Doppler flowmetry; microcirculation; regional anesthesia; sympathetic blockade.

(C) Acta Anaesthesiologica Scandinavica 46 (2002)
$\mathrm{T}$ HE COMBINATION of laser Doppler skin blood flowmetry and finger blood pressure monitoring allows non-invasive assessment of the local vasomotor regulation (1-3). Further, by using this method active and passive changes in local blood perfusion can reliably be distinguished (3). Skin disorders such as painful peripheral nerve injuries (reflex sympathetic dystrophy), ischemia resulting from atherosclerosis, and connective tissue disorders cause altered regulation of the microcirculation of the skin (4-9). Sympathetic blockade can play an important role in the treatment of many of these medical conditions, and may be advantageous after skin transplantation (10-15). However, accurate monitoring of the efficacy of sympathetic blockade is not yet fully developed. We undertook the present study to develop a novel method to monitor the effectiveness of sympathetic blockade. Our proposed method is based on the as- sessment of a baroreflex stimulus-induced skin microcirculatory response.

\section{Methods}

\section{Study population}

The study group consisted of 11 patients (six females, age ranging from 19 to 52 years) who underwent below wrist hand surgery. Their mean height was $169.1 \pm 14.2 \mathrm{~cm}$ and the mean weight was $78.2 \pm 11.4 \mathrm{~kg}$. Only patients with no history of cardiovascular disease, showing no abnormalities when physically examined, and a normal 12-lead ECG were selected for this study. Patients with any other medical condition likely to affect baroreceptor regulation were excluded. All patients were ASA I, and none had taken any medication. All investigations were performed in 


\section{T. Szili-Torok et al.}

an ambient room temperature of $21-22^{\circ} \mathrm{C}$. The subjects were informed about the risks and benefits of this institutionally approved study before providing their written consent. Blood pressure (BP), heart rate (HR), skin blood flow (SBF), skin temperature (SkT), respiratory frequency and respiratory depth were continuously monitored.

\section{Study protocol}

As a routine analgesic procedure for below-wrist hand surgery, axillary brachial plexus block was performed by inserting a 22-G, 5-cm needle and injecting $30 \mathrm{ml}$ lidocain $(1 \%)$ and $30 \mathrm{ml}$ bupivacain $(0.25 \%)$. To locate the plexus a nerve stimulator was used (Stimuplex, Braun, Germany). The position of the needle was judged adequate when an output lower than $0.5 \mathrm{~mA}$ still elicited a slight distal motor response. Sension block and motor block of musculocutaneous, radial, ulnar and median nerve were recorded in every $5 \mathrm{~min}$. Sensory onset of each nerve was assessed by pinprick. Motor block was evaluated with thumb abduction, thumb opposition and elbow flexion. If a surgical level of anesthesia was not reached the patient was not included into the laser Doppler measurements. Local infiltration of the operation area was not necessary in any of the patients during the surgical procedure.

For skin microvascular response evaluation, the subjects were studied in a supine rest position. The subjects were instrumented for the measurement of HR, finger BP, SkT and SBF. For our recordings, the study was not started until the stable baseline of BP, HR and SBF signals for each subject had been obtained. After instrumentation and 5-min baseline data collection patients were requested to perform a Valsalva maneuver.

\section{Measurement of skin blood flow}

Relative blood cell perfusion was measured with a double-channel skin perfusion monitor (Periflux 4001, Perimed, Stockholm, Sweden). This method uses the frequency shift of laser light (2-mW helium-neon laser source of 632.8-nm wavelength) induced by reflection on moving red blood cells to measure red blood cell flux. Skin laser Doppler flow values cannot be expressed in conventional physiological units unless certain conditions are fulfilled. In vivo the readings are therefore expressed in perfusion units related to the Brownian motion in motility standard emulsion provided by the manufacturer. At standard temperature the emulsion produces a motility of 250 perfusion units. This corresponds with $2.5 \mathrm{~V}$ analog perfusion output. The measurement field of the laser Doppler skin probe is restricted to a 1-mm (3) area of the skin microvasculature.

The laser Doppler probes and probe holders were attached to the ventral side of the treated forearm and the control site was at a corresponding area on the untreated arm. The SkT probe was placed close to the laser Doppler probe holders, on both sites.

\section{Continuous blood pressure and ECG monitoring}

The BP was measured continuously with a non-invasive BP monitor developed by Wesseling and coworkers (Finapres 2300, Ohmeda). The measurement is based on the Penaz principle. The ECG was continuously monitored (Sirecust 730, Siemens). The same device provides an impedance signal to monitor the depth and frequency of respiration. The $\mathrm{R}$ wave of the ECG and the plethysmographic signals were fed through an amplifier, filter and analog-digital converter into an IBM-AT compatible computer. Data were stored and analyzed off-line by a program developed in our laboratory written in Microsoft C/ $\mathrm{C}++7.0$. The computer measured the interval between successive $R$ waves with a precision of $2 \mathrm{~ms}$. The accuracy of BP signal detection was 40 microvolts and $1 \mathrm{mmHg}$. The cardiotachogram and trend grams of the BP and SBF values were continuously recorded, and analyzed off-line.

\section{Valsalva maneuver}

Each subject was asked to maintain a column of mercury at $40-50 \mathrm{mmHg}$ for $15 \mathrm{~s}$ with forced expiration, and then to resume normal expiration. A small air leakage was allowed to prevent closure of the glottis. During the test three characteristic points were selected (V1, V2, V3) for further assessment. V1 is the early strain, V2 is the late strain and V3 indicates the overshoot phase of the Valsalva maneuver (Fig. 1).

\section{Calculation of the cutaneous vascular resistance}

Calculation of the cutaneous vascular resistance (CVR) was determined as the ratio of mean arterial pressure (MAP) and skin blood flow (SBF). The percentage change in cutaneous vascular resistance (dCVR) was expressed as follows:

$\mathrm{dCVR}=($ CVRx - CVRbase $) /$ CVRbase $(1), \quad$ where $\times$ denotes the characteristic points (V1, V2, V3) of the test.

\section{Statistical analysis}

Continuous variables are expressed as mean \pm standard deviation. Normally distributed data were compared using the one-way repeated measurement ANOVA (i.e. MAP, HR, SBF). To isolate which 
group differed from the others we used a multiple comparison procedure. (Bonferroni $t$-test). Nongaussian data were analyzed using the Friedman repeated measurement ANOVA on ranks test (i.e. CVR). Student's $t$-test and Newman-Keuls method performed all pair-wise multiple comparisons. The level of significance was set at a $P$-value of 0.05 .

\section{Results}

Reflexes were studied at constant ambient temperature. The range of SkT of the patients was very narrow, both of the treated and non-treated skin regions $\left(33.6 \pm 0.9^{\circ} \mathrm{C} ; 32.9 \pm 0.7^{\circ} \mathrm{C}\right.$, respectively). No significant

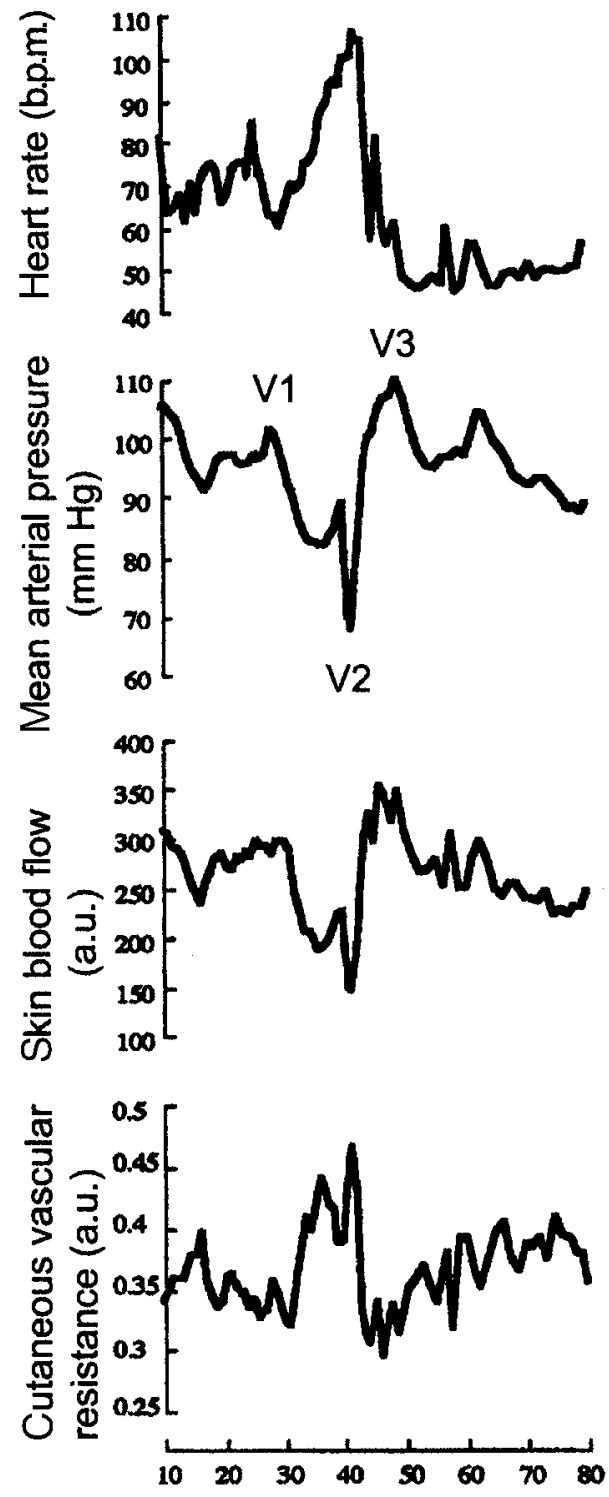

Fig.1. Typical hemodynamic and skin microvascular responses to the Valsalva maneuver, where V1,V2 and V3 indicate the characteristic points selected for the measurements. fluctuations of SkT were detected during any of the short-term reflex tests.

\section{Comparison of the baseline skin blood flow and} cutaneous vascular resistance

There was no significant difference in SBF and CVR values between the two sides before the axillary blockade was performed (SBF: axillary blockade side $534 \pm 195$ a.u., control side: $559 \pm 215$ a.u., $P=$ NS; CVR: axillary side $0.16 \pm 0.8$, control side $0.17 \pm 0.2$ a.u., $P=$ NS) After the axillary blockade became effective SBF and CVR did not show significant change between the treated and control regions (SBF: axillary blockade side $636 \pm 272$ a.u., control side: $547 \pm 197$ a.u., $P=$ NS; CVR: axillary side $0.16 \pm 0.9$, control side $0.17 \pm 0.1$ relative units, $P=\mathrm{NS}$ ). Skin blood flow on the side of the axillary blockade showed a tendency to increase, but CVR did not change after axillary blockade.

Valsalva maneuver-induced responses at the control area (Table 1)

The typical HR, BP and SBF responses to Valsalva maneuver are shown in Figure 1. Skin blood flow followed a pattern of four phases corresponding to the four blood pressure phases of the Valsalva maneuver. The initial phase of the Valsalva maneuver (V1 point) was accompanied by a significant increase in MAP, however, the calculated regional peripheral resistance (CVR) remained unchanged. Towards the end of the strain phase (V2 point) there was a drop in MAP below baseline. At this point a marked decrease in SBF was also observed. The calculated CVR showed significant elevation. On release of the Valsalva maneuver (V3 point) significant BP and SBF values were observed. There was no significant change in CVR in this phase of the Valsalva maneuver.

Valsalva maneuver-induced skin vascular responses at the side of the axillary blockade (Table 2)

Skin blood flow and the CVR remained unchanged throughout the maneuver. Towards the end of the strain phase (V2 point) there was a drop in MAP below baseline. At this point there was no observable change in SBF. Therefore, the calculated CVR did not show significant change. In the overshoot phase (V3 point) SBF and CVR remained unchanged.

\section{Discussion}

The principal finding of our study is that sympathetic blockade noticeably modifies the baroreflex stimulusinduced skin microcirculatory responses. This supports the hypothesis that the human skin is involved 


\section{T. Szili-Torok et al.}

in baroreflex regulation under thermoneutral conditions. In addition, measurement of the differences in cutaneous reflex responses after sympathetic blockade between treated and normal regions seems to be a feasible method for clinically evaluating the effectiveness of sympathetic blockade.

\section{Baroreflex regulation of the human skin microcirculation}

The importance of this subject stems from animal studies, which demonstrated that vessels of the skin could be targets for important powerful homeostatic baroreceptor reflexes (16). On the other hand, in humans the concept that arterial baroreceptors have only a minor influence on limb vascular resistance was developed $(17,18)$. In contrast to these data, Edfeldt and colleagues have demonstrated in their studies using lower body negative pressure that deactivation of the arterial baroreceptors significantly contributes to the regulation of forearm resistance, which involves active participation of the skin microvasculature (19-21). In a recent study, Bernardi and colleagues reported that laser Doppler flowmetry fluctuations were responsive to sinusoidal neck suction, indicating a response to sympathetic modulation. Thus the changes in SBF reflect the modifications determined by autonomic activity (22). In our present study the Valsalva maneuver was used to stimulate the arterial baroreceptor system. Direct microneurographic methods proved that the second phase of the Valsalva maneuver is accompanied by increased sympathetic activation $(5,23)$. We found that the Valsalva maneuver induces a significant change in CVR during the strain phase. This indicates that an active mechanism is participating in this change. The above findings lend support to previous reports suggesting that sympathetic vasomotor fibers of the human skin microvasculature can exert a very potent control of vascular resistance under thermoneutral conditions. This control must partly ascribe to reflexes originating from the low-pressure cardiopulmonary receptors, however, indirect evidence suggests that the high-pressure arterial receptor system also contributes to a significant extent.

\section{Clinical assessment of the skin microcirculation and methodological problems}

Although sympathetic blockade is widely used in clinical practice, the reliable assessment of its efficacy has not yet been developed. Previously the temperature of the treated region was assessed by physical examination. In contrast with this daily routine, Sherman et al. have demonstrated elegantly that the painful limb can be cooler but sometimes warmer than the other limb (6). Furthermore, the relative coolness is not proportional to the pain intensity (6). There is also inconsistency between pain localization and the greatest thermal asymmetry assessed by video thermography

\section{Table 1}

Mean arterial pressure, skin blood flow and cutaneous vascular resistance at the three characteristic points of the Valsalva maneuver at the control site.

\begin{tabular}{lcccc}
\hline & Baseline & V1 & V2 & V3 \\
\hline MAP (mmHg) & $77.5 \pm 8.3$ & $104.7 \pm 9.2^{*}$ & $65.4 \pm 9.1^{*}$ & $122.2^{*} \pm 12.4^{*}$ \\
SBF (a.u) & $546.5 \pm 272.2$ & $655.1 \pm 222.62$ & $314.9 \pm 154.2^{*}$ & $722.2 \pm 195.2^{*}$ \\
CVR (a.u) & $0.18 \pm 0.1$ & $0.17 \pm 0.1$ & $0.42 \pm 0.2^{*}$ & $0.17 \pm 0.2$ \\
\hline
\end{tabular}

MAP, mean arterial pressure; SBF, skin blood flow; CVR, cutaneous vascular resistance; V1, V2, V3 are characteristic points of the Valsalva maneuver (explanation in the text); a.u., arbitrary unit.

${ }^{*} P<0.05$ vs. baseline.

Table 2

Mean arterial pressure, skin blood flow and cutaneous vascular resistance at the three characteristic points of the Valsalva maneuver at the site of the axillary blockade.

\begin{tabular}{lcccc}
\hline & Baseline & V1 & V2 & V3 \\
\hline MAP (mmHg) & $77.5 \pm 8.3$ & $104.7 \pm 9.2^{*}$ & $65.4 \pm 9.1^{*}$ & $122.2 \pm 12.4^{*}$ \\
SBF (a.u) & $511.5 \pm 294.6$ & $698.1 \pm 322.62$ & $415.9 \pm 134.3$ & $589.2 \pm 199.9$ \\
CVR (a.u) & $0.17 \pm 0.8$ & $0.15 \pm 0.1$ & $0.16 \pm 0.2$ & $0.17 \pm 0.2$ \\
\hline
\end{tabular}

MAP, mean arterial pressure; SBF, skin blood flow; CVR, cutaneous vascular resistance; V1, V2, V3 are characteristic points of the Valsalva maneuver (explanation in the text); a.u., arbitrary unit.

${ }^{*} P<0.05$ vs. baseline. 
$(6,7)$. Oberg and Nilsson reported a technique that uses the frequency shift of the laser light (24-27). Laser Doppler flowmetry is a feasible and non-invasive method for measuring skin perfusion (25). Recently, numerous papers have reported the feasibility of laser Doppler flowmetry in measuring SBF after sympathetic blockade $(11-13,28,29)$. Increased SBF was reported after sympathetic blockade using laser Doppler flowmetry. Established physiological stimuli such as the inspiratory gasp vasoconstrictive response or the cold pressor test have been used to initiate skin vasomotor responses and thereby evaluate sympathetic activity. Several investigators have previously suggested that cutaneous vasoconstrictor reflexes may be useful in the assessment of sympathetic blockade following regional anesthesia (28-31). For similar purposes, a novel system known as the laser Doppler perfusion imager can be used (32). Although the absolute blood flow increased after sympathectomy in these studies, this method is accurate only for short-term monitoring because the laser Doppler method is very sensitive to external stimuli, such as thermal change (33). More importantly, there are a large number of factors such as signal processing, choice of bandwidth, motion artefacts, and instrument calibration that can seriously affect the interpretation of laser Doppler signals (33). These factors are often not considered during routine use. The detection and interpretation of the signal are even more difficult in a routine clinical setting. The decreased adrenergic tone can be compensated by some other factors, including the release of local vasoconstrictor factors such as several forms of endothelins $(34,35)$. The cutaneous cholinergic vasodilator system could also be deactivated by axillary blockade resulting in a new set point in the sympatho-cholinergic balance $(4,35,36)$. To avoid the above-mentioned possible pitfalls of measuring flow values we postulated that the assessment of a reflex response may provide more sophisticated monitoring. As the cutaneous microvasculature is involved in reflex regulation, we tested the responsiveness of the adrenergic vasoconstrictor fibers to baroreflex stimuli. Our study's most unique observation is that responses to the baroreflex stimuli at the side of the blockade and at the control side are basically different.

In conclusion, the human skin microvasculature is involved in baroreflex regulation under thermoneutral conditions. The measurement of a baroreflex-induced skin adrenergic response is feasible to monitor the effectiveness of sympathetic blockade. In addition, in this study, the measurement of blood flow was less reliable for assessing sympathetic blockade than for evaluating reflex responses.

\section{References}

1. Low PA, Neumann C, Dyck PJ, Fealey RD, Tuck RR. Evaluation of skin vasomotor reflexes by using laser Doppler velocimetry. Mayo Clin Proc 1983: 58: 583-592.

2. Westerman RA, Widdop RE, Hannaford J, Low A, Roberts RG, Kent $P$ et al. Laser Doppler velocimetry in the measurement of neurovascular function. Australas Phys Eng Sci Med 1988: 11: 53-66.

3. Torok T, Bari F, Paprika D, Rudas L, Kardos A, Gingl Z. Short-term monitoring of the vascular resistance of the human skin microvasculature. Acta Physiol Hung 1997: 85: 153162.

4. Kovacs L, Torok T, Bari F, Keri Z, Kovacs A, Makula E et al. Impaired microvascular response to cholinergic stimuli in primary Sjogren's syndrome. Ann Rheum Dis 2000: 59: 4853.

5. Pryor SL, Lewis SF, Haller RG, Bertocci LA, Victor RG. Impairment of sympathetic activation during static exercise in patients with muscle phosphorylase deficiency (McArdle's disease). J Clin Invest 1990: 85: 1444-1449.

6. Sherman RA, Karstetter KW, Damiano M, Evans CB. Stability of temperature asymmetries in reflex sympathetic dystrophy over time and changes in pain. Clin J Pain 1994: 10: 71-77.

7. Sherman RA, Woerman AL, Karstetter KW. Comparative effectiveness of videothermography, contact thermography, and infrared beam thermography for scanning relative skin temperature. J Rehabil Res Dev 1996: 33: 377-386.

8. van Dielen FM, Kurvers HA, Dammers R, oude Egbrink MG, Slaaf DW, Tordoir JH et al. Effects of surgical sympathectomy on skin blood flow in a rat model of chronic limb ischemia. World J Surg 1998: 22: 807-811.

9. Kokhan EP, Pinchuk OV. Sympathetic tonus and lumbar sympathectomy in arteriosclerosis obliterans of the arteries of the lower extremities. Vestn Khir Im I I Grek 1997, 156: 17-21.

10. Pollock DC, Li Z, Rosencrance E, Krome J, Koman LA, Smith TL. Acute effects of periarterial sympathectomy on the cutaneous microcirculation. J Orthop Res 1997: 15: 408-413.

11. Lantsberg L, Goldman M. Lower limb sympathectomy assessed by laser Doppler blood flow and transcutaneous oxygen measurements. J Med Eng Technol 1990: 14: 182-183.

12. Lantsberg L, Goldman M. Laser Doppler flowmetry, transcutaneous oxygen tension measurements and Doppler pressure compared in patients undergoing amputation. Eur J Vasc Surg 1991: 5: 195-197.

13. Lantsberg L, Goldman M, Khoda J. Should chemical sympathectomy precede below knee amputation? Int Surg 1996: 81: 85-87.

14. Cooke ED, Harris J, Fleming CE, Steinberg MD, Foster JM. Correlation of pain with temperature and blood-flow changes in the lower limb following chemical lumbar sympathectomy in reflex sympathetic dystrophy. A case report. Int Angiol 1995: 14: 226-228.

15. Barisoni DM, Veall N. Effect of thymoxamine on circulation in skin flaps and in denervated skin. Lancet 1969: 1: 400-401.

16. Shepherd JT, ed. Circulation to Skeletal Muscle. Bethesda, Maryland: American Physiological Society, 1983.

17. Walker JL, Abboud FM, Mark AL, Thames MD. Interaction of cardiopulmonary and somatic reflexes in humans. J Clin Invest 1980: 65: 1491-1497.

18. Mancia G, Mark AL, eds. Arterial Baroreflexes in Humans. Bethesda, Maryland: American Physiological Society, 1983.

19. Lundvall J, Edfeldt H. Sympathetic control of vascular resistance in skeletal muscle and skin of man. Acta Physiol Scand 1991: 143: 359-360. 


\section{T. Szili-Torok et al.}

20. Lundvall J, Edfeldt H. Much more potent baroreflex sympathetic control of vascular resistance in the resting human limb than previously believed. Acta Physiol Scand 1993: 147: 185-193.

21. Edfeldt H, Lundvall J. Sympathetic baroreflex control of vascular resistance in comfortably warm man. Analyses of neurogenic constrictor responses in the resting forearm and in its separate skeletal muscle and skin tissue compartments. Acta Physiol Scand 1993: 147: 437-447.

22. Bernardi L, Hayoz D, Wenzel R, Passino C, Calciati A, Weber $\mathrm{R}$ et al. Synchronous and baroceptor-sensitive oscillations in skin microcirculation: evidence for central autonomic control. Am J Physiol 1997: 273: H1867-H1878.

23. Matsukawa T, Sugiyama Y, Watanabe T, Kobayashi F, Mano T. Baroreflex control of muscle sympathetic nerve activity is attenuated in the elderly. J Auton Nerv Syst 1998: 73: 182185.

24. Oberg PA, Nilsson GE, Tenland T, Holmstrom A, Lewis DH. Use of a new laser Doppler flowmeter for measurement of capillary blood flow in skeletal muscle after bullet wounding. Acta Chir Scand Suppl 1979: 489: 145-150.

25. Nilsson GE, Tenland T, Obert PA. A new instrument for continuous measurement of tissue blood flow by light beating spectroscopy. IEEE Trans Biomed Eng 1980: 27: 12-19.

26. Almond NE, Jones DP, Cooke ED. Noninvasive measurement of the human peripheral circulation: relationship between laser Doppler flowmeter and photoplethysmograph signals from the finger. Angiology 1988: 39: 819-829.

27. Almond NE, Jones DP, Cooke ED. High quality photoplethysmograph signals from a laser Doppler flowmeter: preliminary studies of two simultaneous outputs from the finger. J Biomed Eng 1988: 10: 458-462.

28. Valley MA, Bourke DL, Hamill MP, Raja SN. Time course of sympathetic blockade during epidural anaesthesia: laser Doppler flowmetry studies of regional skin perfusion. Anesth Analg 1993: 76: 289-294.

29. Valley MA, Bourke DL, McKenzie AM, Raja SN. Quantitat- ive testing of sympathetic function with laser Doppler flowmetry. J Clin Monit 1993: 9: 252-256.

30. Netten PM, Wollersheim H, Gielen MJ, Den Arend JA, Lutterman JA, Thien T. The influence of ulnar nerve blockade on skin microvascular blood flow. Eur J Clin Invest 1995: 25: 515-522.

31. Lehtipalo S, Winso O, Koskinen LO, Johansson G, Biber B. Cutaneous sympathetic vasoconstrictor reflexes for the evaluation of interscalene brachial plexus block. Acta Anaesthesiol Scand 2000: 44: 946-952.

32. Sorensen J, Bengtsson M, Malmqvist EL, Nilsson G, Sjoberg F. Laser Doppler perfusion imager (LDPI) - for the assessment of skin blood flow changes following sympathetic blocks. Acta Anaesthesiol Scand 1996: 40: 1145-1148.

33. Obeid AN, Barnett NJ, Dougherty G, Ward G. A critical review of laser Doppler flowmetry. J Med Eng Technol 1990: 14: 178-181.

34. Bull HA, Dowd PM. Edothelin-1 in human skin. Dermatology 1993: 187: 1-5.

35. Burnstock G, Ralevic V. New insights into the local regulation of blood flow by perivascular nerves and endothelium. Br J Plast Surg 1994: 47: 527-543.

36. Torok T, Bari F, Kardos A, Paprika D, Rudas L. Isometric handgrip exercise-induced muscarinic vasodilation in the human skin microvasculature. Acta Physiol Hung 1997: 85: 193-198.

Address:

Tamas Szili-Torok

Department of Clinical Electrophysiology

Thoraxcentre

Rotterdam

Molewaterplein 40

3015G Rotterdam

the Netherlands

e-mail: szili@card.azr.nl 